\title{
A educação dos cuidadores
}

Sofia Madureira*

\section{RESUMO}

Nos últimos anos, em particular na última década, tem-se assistido a um interesse cada vez maior pelo estudo sobre o cuidador informal do doente com demência e sobre a sua importância na melhoria da qualidade de vida e no adiamento da institucionalização do doente. A importância do papel do cuidador e as características associadas ao acto de cuidar, nomeadamente a sobrecarga objectiva e subjectiva, o stress psicológico e os aspectos positivos do acto de cuidar têm sido identificados e descritos. No entanto, a educação dos cuidadores informais é relativamente recente e ainda pouco estruturada, sobretudo no nosso país. Abordamos aqui os aspectos que consideramos importantes na educação do cuidador e no papel do clínico para a sua promoção em consulta. As alterações comportamentais dos doentes são os factores de stress psicológico que apresentam maior sobrecarga para os cuidadores. A investigação aponta para a importância de um conjunto de estratégias de educação para a) o cuidado do doente, e b) o auto-cuidado do próprio cuidador. Salienta-se o papel da auto-eficácia percebida do cuidador como mediador entre os stressores psicológicos e a sintomatologia depressiva. Reforça-se ainda o papel do cuidador como parceiro activo, quer na prestação de cuidados básicos, quer na implementação e adequação de terapêuticas não-farmacológicas.

Palavras-chave: Cuidadores; Demência; Educação.

\section{INTRODUÇÃO}

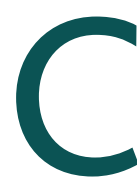

uidador é uma palavra cada vez mais utilizada em associação com as demências e um conceito que engloba a ideia de alguém que toma conta, que trata, que cuida em todos os sentidos. Os cuidadores são tão antigos como a doença, qualquer que ela seja. Apesar disso, a importância que lhes foi dada ao longo do tempo não foi proporcional, tendo a doença ocupado um lugar privilegiado em relação ao cuidador. $O$ aumento da esperança média de vida, a consequente identificação das patologias associadas ao envelhecimento e a avaliação do seu impacto a nível social e económico, obrigaram a uma abordagem multi e interdisciplinar no tratamento da doença e a um olhar diferente sobre o papel do cuidador. Tornou-se mais claro o papel deste agente no bem-estar físico, psicológico e emocional do doente.

Cuidadores são as pessoas que cuidam, que tratam, que apoiam e amparam. Deste grupo fazem parte todos os que, por inerência da profissão ou das actividades escolhidas (enfermeiros, auxiliares de acção médica, assistentes em residências, voluntários, etc.), prestam cuidados directos aos doentes - cuidadores formais -, mas também os que, não o tendo escolhido, se

*Psicóloga Clínica, Mestre em Neurociências, Instituto de Medicina Molecular, Faculdade de Medicina de Lisboa tornam agentes na prestação de cuidados (directos ou indirectos) - os cuidadores informais.

Nas últimas décadas tem sido dado cada vez mais relevo à investigação sobre cuidadores de doentes com demência. ${ }^{1}$ Ao longo de todo o processo da evolução da doença, o papel do(s) cuidador(es) é fundamental. Como noutras situações de doença mental, em que o cuidador assume a prestação de cuidados informais, na demência o cuidador torna-se imprescindível quer como parceiro no tratamento - através da identificação das características das diferentes fases e consequente adaptação terapêutica -, quer como garante da manutenção da qualidade de vida dos doentes. A presença do cuidador informal está directamente relacionada com uma maior permanência em casa e atraso na institucionalização, o que representa uma diminuição considerável nos gastos em cuidados formais. Por este motivo, a identificação das necessidades dos cuidadores e a implementação de programas de apoio para estes tornam-se objectivos cada vez mais pertinentes.

De acordo com os dados da associação Alzheimer Portugal, estima-se que em Portugal existam 153.000 pessoas com demência, 90.000 das quais com doença de Alzheimer. Embora não se saiba ao certo o número de cuidadores por doente (podendo haver mais do que um por paciente) e qual o número de doentes em ins- 
tituição em Portugal, estudos realizados noutros países estimam que cerca de $75 \%$ a $80 \%$ dos doentes de Alzheimer estarão em suas casas, assistidos maioritariamente pelos seus familiares. ${ }^{2-3}$

\section{ALGUNS DADOS SOBRE OS CUIDADORES}

Um dos achados mais importantes na investigação sobre os cuidadores de doentes com demência foi o reconhecimento do impacto da doença em quem cuida, nomeadamente das consequências na sua saúde mental e física. Os primeiros trabalhos nesta área mostraram que os cuidadores informais (e formais) dos doentes com demência apresentam maior risco de desenvolver patologia física e psicológica. ${ }^{4-5}$ Apresentam igualmente um maior risco de morbilidade somática (hipertensão arterial, hiperlipidemia, alterações imunológicas) e até de mortalidade quando se trata de cuidadores mais idosos. ${ }^{6}$

Sabe-se também que a proporção de cuidadores com morbilidade psiquiátrica é superior à da população em geral. A depressão e ansiedade são as perturbações mais frequentes, variando a depressão geral entre $30 \%$ a $70 \%{ }^{7}$ e a ansiedade entre $6 \%$ a $25 \% .^{8}$

As alterações psicopatológicas podem estar relacionadas com uma série de factores que vão desde a resposta adaptativa às perdas sucessivas - do que é conhecido no familiar, uma vez que a demência se caracteriza também pela perda do "self”; da relação prévia; dos papéis pré-determinados - da sobrecarga subjectiva, até à própria vulnerabilidade do cuidador - idade mais avançada, presença de outras doenças e diminuição do tempo e disponibilidade para o auto-cuidado. ${ }^{7}$

Nos países ocidentais, $30 \%$ a $60 \%$ dos cuidadores informais são os respectivos cônjuges, sendo a proporção de mulheres de 2/3 a 3/4. Nos países mediterrânicos, as mulheres, mais do que os maridos, ocupam o lugar de cuidador principal - aquele que providencia mais tempo na prestação de cuidados -, estando mais próximas emocional e fisicamente. ${ }^{7}$ A maior parte dos cuidadores dos doentes com demência são as mulheres (cônjuges ou filhas), o que parece decorrer do prolongamento dos papéis tradicionais de mulher/filha. O lugar de cuidador principal acaba, muitas vezes, por ser estabelecido pela continuidade das tarefas, sem que haja uma preparação ou sequer uma escolha prévias. Muitas vezes é a proximidade física e emocional do doente no momento do diagnóstico que determina o lugar de cuidador principal, sem que haja uma compreensão clara das funções a desempenhar.

$\mathrm{O}$ acto de cuidar, em particular de um doente com demência, traz associada uma sobrecarga física, emocional e psíquica, mas também pode trazer factores positivos, como por exemplo, gratificação pessoal e sentido para a vida. ${ }^{7,9-10}$ A manutenção do equilíbrio entre estas duas componentes representa um desafio para o cuidador (informal ou formal), para a família e para os técnicos que os acompanham. A estes últimos acresce a responsabilidade de identificar os factores de risco para o aumento da sobrecarga (factores relacionados com a própria doença, com as consequências da prestação dos cuidados ou com as características do próprio cuidador), as necessidades dos cuidadores e a determinação de terapêuticas para o seu re-equilíbrio, o qual passa essencialmente pela educação dos cuidadores.

\section{O PAPEL DO CUIDADOR E A EDUCAÇÃO PARA O SEU DESEMPENHO}

Ao contrário de muitos outros papéis assumidos ao longo da vida, para o de cuidador informal de um doente com demência não há uma preparação prévia. A confirmação do diagnóstico, muitas vezes temido mas quase sempre surpreendente, marca o momento de passagem de um qualquer papel - tipicamente o de mulher/companheira ou filha - ao de cuidador principal.

Neste papel está incluída uma variedade de funções, que vão desde as menos exigentes - como conduzir, pagar contas, gerir rotinas -, às que exigem maior esforço físico - lavar, vestir, alimentar - e psíquico - controlo das alterações comportamentais, supervisão constante -, sendo as últimas as mais responsáveis por um risco acrescido para a sobrecarga. ${ }^{7}$ As funções, bem como o tempo exigido na prestação de cuidados, vão aumentando à medida que a doença progride, criando níveis de sobrecarga objectiva e subjectiva directamente proporcionais, até ao momento da institucionalização, altura em que há uma diminuição da sobrecarga objectiva embora nem sempre acompanhada da redução dos factores de stress psicológico. ${ }^{7}$

\section{A EDUCAÇÃO DO CUIDADOR INFORMAL}

Sabe-se que a relação cuidador/doente se reveste de características próprias como a presença de sobrecarga 


QUADRO I. «Regra de três» na passagem de informação
Na altura do diagnóstico
\begin{tabular}{l|l|l|l|}
\hline $\begin{array}{l}\text { Numa consulta de } \\
\text { seguimento precoce }\end{array}$ & $\begin{array}{l}\text { Numa consulta de } \\
\text { seguimento mais tardia }\end{array}$ & $\begin{array}{l}\text { Numa consulta de } \\
\text { seguimento ainda mais tardia }\end{array}$ \\
\hline O que é a demência & Serviços disponíveis & Grupos de suporte & Psicoterapias \\
\hline Medicamentos disponíveis & Evolução da doença & Benefícios & Repercussões nos cuidadores \\
\hline Alterações comportamentais e & O que fazer numa crise & Questões financeiras e legais & Terapias complementares \\
\hline
\end{tabular}

Adaptado por: Gonçalves-Pereira e Mateos ${ }^{7}$

objectiva e subjectiva, o stress psicológico e, mais recentemente identificados, os aspectos positivos associados ao acto de cuidar. ${ }^{10}$ Sabe-se também que esta relação é mediada por uma multiplicidade de factores que vão desde as características do doente e do cuidador (idade, sexo, personalidade prévia), à existência de redes sociais de suporte, estratégias de coping e diferenças étnicas/culturais, passando pela qualidade e tipo de relação prévia e actual entre os dois. ${ }^{7}$

Tendo em conta todos estes aspectos relacionados com a especificidade da relação cuidador/doente e a dificuldade que eles acarretam para uma avaliação eficaz das necessidades, a educação do cuidador deve integrar duas áreas fundamentais:

a) A de cuidar do outro: com a informação clara acerca do diagnóstico e prognóstico e o desenvolvimento de conhecimentos, estratégias e treino de competências na prestação directa de cuidados ao doente; b) A de cuidar de si próprio: com o treino de promoção da auto-eficácia percebida e reconhecimento dos seus limites, fomentação de estratégias de resiliência e de auto-cuidado.

Em relação ao primeiro ponto, salientam-se os aspectos relacionados com:

- Comunicação do diagnóstico: a informação acerca do diagnóstico deve contemplar a passagem clara de informação, numa linguagem adaptada ao nível cultural/educacional do cuidador. ${ }^{11,12}$ Transmitir um diagnóstico de demência é dar uma má notícia e deve ser encarado como o primeiro confronto com uma realidade muitas vezes esperada, mas não desejada. Este aspecto torna-se tanto mais pertinente quanto mais precoce é o diagnóstico. O conhecimento que o cuidador tem acerca da doença influencia a sua percepção, o modo como reage e a forma como se organiza na resposta às suas necessidades e às do doente..$^{13}$ No entanto, a forma como os cuidadores têm acesso à informação e o modo como esta se transforma em conhecimento prático é variável e nem sempre claro. Para além da transmissão da informação, é fundamental avaliar o modo como essa informação chega ao interlocutor (feedback) e o que sabe para além do que lhe foi dito. Embora exija uma maior disponibilidade por parte do técnico, é uma forma directa de avaliar a capacidade de compreensão e grau de conhecimento acerca da demência. De acordo com Wald, as necessidades de informação por parte dos cuidadores diferem de consulta para consulta. ${ }^{14}$ Gonçalves-Pereira ilustra, de forma clara (Quadro 1), a «regra de três» utilizada por Wald, para definir as três categorias da informação mais pertinente em cada consulta.

- Informação acerca da doença: tem sido apontada uma relação entre o conhecimento acerca da doença e melhores respostas adaptativas por parte dos cuidadores ${ }^{13}$ e níveis mais baixos de depressão. ${ }^{15}$ No entanto, a informação per senão é suficiente para diminuir os níveis de sobrecarga do cuidador ou melhorar o seu bem-estar. ${ }^{16}$ Num estudo recente, Gonçalves-Pereira e col. avaliaram o grau de conhecimento acerca da doença relacionando-o com as experiências dos cuidadores. ${ }^{17}$ Embora os 116 cuidadores observados tivessem um bom nível de conhecimento da doença, quase metade apresentava morbilidade psicopatológica (depressiva /ansiosa) directamente relacionada com as medidas de sobrecarga. A informação dada, bem como as estratégias de educação para a saúde, devem ser inseridas 
em programas de intervenção mais completos, que englobem abordagens psicoeducativas.

No que se refere à educação sobre cuidar de si próprio, incluem-se:

- Avaliação do cuidador pelo técnico: tem-se revelado importante a identificação dos factores de risco para sobrecarga (e.g. ausência de suporte social) e stress psicológico (e.g. estratégias de coping desadequadas). Esta avaliação pode ser feita através da utilização de instrumentos específicos (Escala de Sobrecarga de Zarit, ${ }^{18}$ por exemplo, disponível em português, no livro de escalas de demência, ${ }^{19}$ ou através da entrevista clínica não estruturada);

- Informação sobre o impacto e repercussão da doença na saúde física e mental dos cuidadores: salientam-se os aspectos negativos e os factores positivos associados ao acto de cuidar (sensação de preenchimento, orgulho em relação a ser capaz de gerir os problemas, gratificação), proporcionando o acesso a informação escrita (biblioterapia) e outros apoios (associações de doentes e/ou familiares);

- Sensibilização do cuidador para pedidos de ajuda e procura de serviços: embora não haja uma diversidade de apoios acessíveis, muitos dos cuidadores não procuram os poucos existentes, quer por falta de informação, quer por ausência de necessidade percebida; ${ }^{317}$

- Apoio e aconselhamento multidisciplinar básicos: procurando responder às necessidades sociais, médicas, educacionais e psicológicas que ocorrem ao longo da evolução da doença; ${ }^{3}$

- Intervenções para a diminuição da morbilidade psicopatológica e melhoria da qualidade de vida do cuidador e, consequentemente, do doente: estas podem ser intervenções de suporte (validação de sentimentos, apoio emocional e partilha de informações), psicoeducativas (eminentemente informativas), de alívio (procuram diminuir de forma directa a sobrecarga do cuidador através de internamentos curtos em instituições, por exemplo) ou psicoterapia individual (de inspiração cognitiva-comportamental ou outra, dependendo dos objectivos); ${ }^{20}$

- Intervenções individuais, orientadas para as necessidades específicas do cuidador: a terapia individual de orientação preferencial cognitiva-comportamental, com treino de estratégias de coping, sobretudo no controlo das alterações comportamentais dos doentes, verifica-se eficaz no alívio dos sintomas e manutenção do bem-estar do cuidador. ${ }^{21} \mathrm{~A}$ promoção de estratégias que reforcem a auto-eficácia ${ }^{22}$ no controlo das alterações comportamentais e de memória do doente, está associada a uma redução da sintomatologia depressiva do cuidador. ${ }^{23} \mathrm{~A}$ escolha do tipo de intervenção (individual vs grupo; psicoeducativa vs de suporte vs combinada com estratégias de resolução de problemas ou modificação de comportamento) deve ser feita de acordo com as necessidades individuais do cuidador;

- Implementação de estratégias de auto-cuidado: a sensibilização para o auto-cuidado é um dos trabalhos de maior relevância para a prevenção de burnout nos cuidadores informais (e formais). A disponibilidade para cuidar associa-se, muitas vezes, à perda da individualidade. Trabalhar sentimentos como a culpabilidade (associada à ideia de que cuidar exige disponibilidade total e consequente desvalorização das necessidades individuais), é um processo que pode ser lento e deve ter em conta as características individuais, educacionais e culturais do cuidador;

- Capacitação (empowerment) do cuidador como parceiro terapêutico: pretende-se ajudar o cuidador a adquirir os instrumentos promotores de controlo sobre a situação (decisão partilhada das terapêuticas escolhidas e adequação ao longo do tempo, por exemplo), reconhecer o cuidador como um dos peritos do processo de evolução da doença, possibilitando-lhe a informação/orientação para intervenções directas com o doente no seu meio (como a implementação de estratégias de orientação para a realidade ou de exercícios de estimulação cognitiva). Muitos cuidadores expressam uma sensação de bem-estar com a participação activa na prestação de cuidados básicos e na introdução de terapêuticas não-farmacológicas, como contraponto ao sentimento de impotência associado à crença de que, não havendo cura, não existe «nada a fazer».

Por fim, a intervenção com o cuidador estará completa com a monitorização da qualidade da relação cuidador/doente ao longo da evolução da doença, uma vez que a adaptação de estratégias eficazes num determinado momento/fase da doença, não garante o seu 
sucesso ao longo da sua evolução. Será útil a avaliação frequente das necessidades dos cuidadores, do tipo de ajuda esperado, das expectativas em relação às intervenções e da forma como aderem às propostas apresentadas. A não adesão às terapêuticas pode passar, com frequência, por problemas de ordem económica, cultural e educacional que devem ser contextualizados.

A avaliação e monitorização das necessidades dos cuidadores no contexto da consulta de Medicina Geral e Familiar implica, na maior parte das vezes, o encaminhamento quer para outras consultas da especialidade existentes no serviço público (Psiquiatria, Psicogeriatria, consulta de Demências), quer para outros serviços, nomeadamente para as associações de doentes/ / familiares (é o caso da Associação Portuguesa dos Familiares e Amigos dos Doentes de Alzheimer), organizadas de forma a complementar ou oferecer apoios específicos (ver Apêndice).

\section{CONCLUSÃO}

A educação do cuidador informal é um processo multidisciplinar (envolve médicos, enfermeiros, psicólogos e assistentes sociais), de longa duração (tem início no momento do diagnóstico e acompanha o tempo de evolução da doença, podendo ir para além da morte do doente) e que exige uma disponibilidade nem sempre possível na organização actual dos serviços públicos. No entanto, o reconhecimento das necessidades destes cuidadores, com a consequente implementação de programas adequados e a monitorização dos seus efeitos, torna-se imprescindível na melhoria da qualidade de vida dos doentes e seus familiares e no atraso da institucionalização.

\section{REFERÊNCIAS BIBLIOGRÁFICAS}

1. Nelis S, Quinn C, L. C. Information and support interventions for informal caregivers of people with dementia (Protocol). Cochrane Database of Systematic Reviews 2007; (2): CD006440.

2. Dunkin JJ, Anderson-Hanley C. Dementia caregiver burden: a review of the literature and guidelines for assessment and intervention. Neurology 1998; 51 (1 Suppl 1): S53-60; discussion S65-7.

3. Rosa E, Lussignoli G, Sabbatini F, Chiappa A, Di Cesare S, Lamanna L, et al. Needs of caregivers of the patients with dementia. Arch Gerontol Geriatr 2009;doi: 10.1016/j.archger.2009.07.008.

4. Brodaty $H$, Hadzi-Pavlovic D. Psychosocial effects on carers of living with persons with dementia. Aust N Z J Psychiat 1990 Sep; 24 (3): 35161.

5. Schultz R, Williamson G. A two-year longitudinal study of depression amongst Alzheimer's care-givers. Alz Dis Assoc Dis 1997; 11: 117-24.

6. Haley WE. The family caregivers role in Alzheimer's Disease. Neurology 1997 May; 48 (6): S25-S29.

7. Goncalves Pereira M, Mateos R. A família e as pessoas com demência: vivências e necessidades dos cuidadores. In: Firmino $\mathrm{H}$, editor. Psicogeriatria. Coimbra: Psiquiatria Única; 2006.

8. Mahoney R, Regan C, Katona C, Livingston G. Anxiety and depression in family caregivers of people with Alzheimer disease: the LASER-AD study. Am J Geriatr Psychiatry 2005 Sep; 13 (9): 795-801.

9. Brodaty $\mathrm{H}, \mathrm{Berman} \mathrm{K}$. Social management of dementia for patients and carers. In: Ritchie CW, Ames D, Masters CL, Cummings JL, editors. Therapeutic strategies in dementia. Oxford: Clinical Publishing; 2007. p. 255-69.

10. Cohen CA, Colantonio A, Vernich L. Positive aspects of caregiving: rounding out the caregiver experience. Int J Geriatr Psychiatry 2002 Feb; 17 (2): 184-8.

11. van den Brink-Muinen A, Verhaak PF, Bensing JM, Bahrs O, Deveugele $M$, Gask L, et al. Doctor-patient communication in different European health care systems: relevance and performance from the patients' perspective. Patient Educ Couns 2000 Jan; 39 (1): 115-27.

12. Silva PR. A comunicação na prática médica: seu papel como componente terapêutico. Rev Port Clin Geral 2008 Jul-Ago; 24 (4): 505-12.

13. McNaughton ME, Patterson TL, Smith TL, Grant I. The relationship among stress, depression, locus of control, irrational beliefs, social support, and health in Alzheimer's disease caregivers. J Nerv Ment Dis 1995 Feb; 183 (2): 78-85.

14. Wald C, Fahy M, Walker Z, Livingston G. What to tell dementia caregivers: the rule of threes. Int J Geriatr Psychiatry 2003 Apr; 18 (4): 313 7.

15. Graham C, Ballard C, Sham P. Carers' knowledge of dementia, their coping strategies and morbidity. Int J Geriatr Psychiatry 1997 Sep; 12 (9): 931-6.

16. Kurz A, Wagenpfeil S, Hallauer J, Schneider-Schelte H, Jansen S. Evaluation of a brief educational program for dementia carers: The AENEAS Study. Int J Geriatr Psychiatry 2009 Nov 27 [epub ahead of print]

17. Goncalves-Pereira M, Carmo I, da Silva JA, Papoila AL, Mateos R, Zarit $\mathrm{SH}$. Caregiving experiences and knowledge about dementia in Portuguese clinical outpatient settings. Int Psychogeriatr 2010 Mar; 22 (2): 270-80.

18. Zarit SH, Todd PA, Zarit JM. Subjective burden of husbands and wives as caregivers: a longitudinal study. Gerontologist 1986 Jun; 26 (3): 2606.

19. Grupo de Estudos de Envelhecimento Cerebral e Demência. Escalas e testes na demência. Lisboa: GEECD; 2008.

20. Sörensen S, Pinquart M, Duberstein P. How effective are interventions with caregivers? An updated meta-analysis. Gerontologist 2002 Jun; 42 (3): 356-72.

21. Selwood A, Johnston K, Katona C, Lyketsos C, Livingston G. Systematic review of the effect of psychological interventions on family caregivers of people with dementia. J Affect Disord 2007 Aug; 101 (1-3): 75-89.

22. Bandura Al. Self-efficacy mechanisms in physiological activation and health promoting behavior. In: Madden J, editor. Neurobiology of Learning, Emotion and Affect. New York: Raven; 1991. p. 229-70. 
23. Rabinowitz YG, Mausbach BT, Gallagher-Thompson D. Self-efficacy as a moderator of the relationship between care recipient memory and behavioral problems and caregiver depression in female dementia caregivers. Alzheimer Dis Assoc Disord 2009 Oct-Dec; 23 (4): 389-94.

\section{ENDEREÇO PARA CORRESPONDÊNCIA}

Sofia Madureira

Hospital de Santa Maria, Serviço de Neurologia - Piso 6,

Av. Prof. Egas Moniz,

1600 Lisboa

A autora declarou não possuir conflitos de interesses

\section{ABSTRACT}

\section{CAREGIVERS EDUCATION}

The importance of informal caregiving with patients with dementia has been enhanced during the last decade, namely in the promotion of quality of life and delay of institutionalized care. Several studies have reported specific characteristics associated with the caregivers' role such as the subjective burden, psychological stress and, more recently, also positive variables related with caregiving. However, there is a lack of formal education of the caregivers, which creates difficulties in the way such role could be performed. We report the most important aspects that should be considered and promoted by the General and Family Medicine practitioner in the education of the caregivers. Caregivers' education should be grounded in two main factors a) taking care of the patient, and b) taking care of him/her-self. In this paper we also report the importance of caregiver perceived self-efficacy mechanisms as mediators between psychological stressors and depressive symptoms. We also reinforce the importance of the caregiver as an active partner of the clinician in the promotion of non-pharmacological strategies, especially in the management of behavioral problems.

Keywords: Caregivers; Dementia; Education.

\section{APÊNDICE}

APFADA - ASSOCIAÇÃO PORTUGUESA DE FAMILIARES E AMIGOS DOS DOENTES DE ALZHEIMER

\section{SEDE}

Av. de Ceuta Norte, Lote 15, Piso 3

Quinta do Loureiro

1300 - 125 Lisboa

Telefones: 213610460 / 8

Fax: 213610469

geral@alzheimerportugal.org

\section{DELEGAÇÃO DO NORTE}

R. Barão do Corvo, 181

4400-039 Vila Nova de Gaia

Telefone: 226066863

Fax: 223754484

geral.norte@alzheimerportugal.org

DELEGAÇÃO CENTRO

Centro de Saúde de Pombal

\author{
3100-000 POMBAL \\ Tel.: 236200970 \\ Fax: 236200971 \\ E-mail: geral.centro@alzheimerportugal.org
}

\section{DELEGAÇÃO DA REGIÃO AUTÓNOMA DA MADEIRA}

Complexo Habitacional da Nazaré, Cave do Bloco 21 - Sala E

9000-135 FUNCHAL

Telefone e Fax: 291772021

E-mail: geral.madeira@alzheimerportugal.org

NÚCLEO DO RIBATEJO

R. Dom Gonçalo da Silveira N. ${ }^{\circ} 31$-A

2080-114 Almeirim

\section{NÚCLEO DE AVEIRO}

Santa Casa da Misericórdia de Aveiro

Complexo Social da Quinta da Moita - Oliveirinha

3810 Aveiro 